\title{
ENERGY GAP STRUCTURE AND TUNNELING CHARACTERISTICS OF LAYERED SUPERCONDUCTORS*
}

\author{
S. H. Liu \\ Solid State Division \\ Oak Ridge National Laboratory \\ Oak Ridge, TN 37831-6032 \\ R. A. Klemm \\ Materials Science Division \\ Argonne National Laboratory \\ Argonne, IL 60439
}
The submitted manuscript has been authored by a contractor of the U. S. Government under contract No. W-31-109-ENG-38. Accordingly, the U.S. Government retains a nonexclusive, royalty-free license to publish or reproduce the published form of this contribution, or allow others to do so, for U.S. Government purposes.

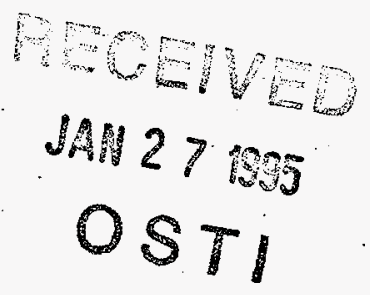

JUNE 1993

/rlp

\section{Distribution: \\ 1-3. M. Masek \\ 4. B. D. Dunlap \\ 5. M. B. Brodsky \\ 6. A. A. Abrikosov \\ 7-8. Authors \\ 9. Editorial Office \\ 10. F. Y. Fradin}

\section{DISCLAIMER}

This report was prepared as an account of work sponsored by an agency of the United States Government. Neither the United States Government nor any agency thereof, nor any of their employees, makes any warranty, express or implied, or assumes any legal liability or responsibility for the accuracy, completeness, or usefulness of any information, apparatus, product, or process disclosed, or represents that its use would not infringe privately owned rights. Reference herein to any specific commercial product, process, or service by trade name, trademark, manufacturer, or otherwise does not necessarily constitute or imply its endorsement, recommendation, or favoring by the United States Government or any agency thereof. The views and opinions of authors expressed herein do not necessarily state or reflect those of the United States Government or any agency thereof.

Submitted Phys. Rev. B.

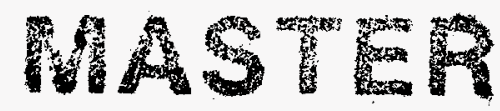

* Work Supported by the U.S. Department of Energy, BES-Materials Sciences, under Contract \#W-31-109-ENG-38. 


\section{DISCLAIMER}

Portions of this document may be illegible in electronic image products. Images are produced from the best available original document. 


\title{
ENERGY GAP STRUCTURE AND TUNNELING CHARACTERISTICS OF LAYERED SUPERCONDUCTORS ${ }^{*}$
}

\author{
S. H. Liu \\ Solid State Division \\ Oak Ridge National Laboratory \\ Oak Ridge, TN 37831-6032 \\ R. A. Klemm \\ Materials Science Division \\ Argonne National Laboratory \\ Argonne, IL 60439
}
The submitted manuscript has been authored by a contractor of the U. S. Government under contract No. W-31-109-ENG-38.
Accordingly, the U.S. Government retains a nonexclusive, royalty-free license to publish or reproduce the published form of this contribution, or allow others to do so, for U.S. Government purposes.

/rlp

JUNE 1993

Submitted Phys. Rev. B.

* Work Supported by the U.S. Department of Energy, BES-Materials Sciences, under Contract \#W-31-109-ENG-38. 


\title{
ENERGY GAP STRUCTURE AND TUNNELING CHARACTERISTICS OF LAYERED SUPERCONDUCTORS
}

\author{
by \\ S. H. Liu \\ Solid State Division, Oak Ridge National Laboratory, Oak Ridge, TN 37831-6032 \\ and
}

R. A. Klemm

Materials Science Division, Argonne National Laboratory, Argonne, IL 60439

\begin{abstract}
We have analyzed the energy gaps and density-of-states (DOS) of layered superconductors with two inequivalent layers in a unit cell along the $c$-axis. In the physically. interesting parameter range where the interlayer hopping strengths of the quasiparticles are comparable to the critical temperature, the peaks in the DOS curve do not correspond to the order parameters (OP's) of each layer, but depend on the OP's and the interlayer hopping strengths in a complex manner. In contrast to a BCS superconductor, the DOS of layered systems have logarithmic singularities. Our simulated tunneling characteristics bear close resemblance to experimental results.
\end{abstract}

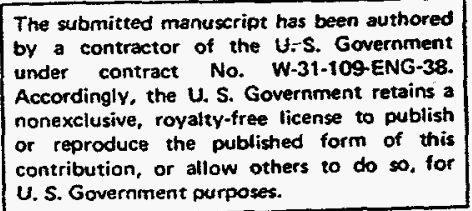




\section{Introduction}

All copper oxide based high- $\mathrm{T}_{c}$ superconductors have stratified structures, in which the quasiparticles move freely within the layers but hop weakly between layers. It has occurred to a number of authors that the nearly two-dimensional geometry may play an important role in the phenomenology of these materials [1-8]. Much of this effort has been stimulated by the tunneling spectroscopy data, which exhibit far more complex structure than low- $\mathrm{T}_{c}$ superconductors. In general, the theories predict that in the weak hopping limit in which the interlayer hopping strengths of the quasiparticles are weak compared with the critical temperature $\mathcal{T}_{c}$, the DOS curves have peaks at the energy gaps or order parameters of individual layers [1-2]. In the strong hopping limit, however, the main features in the DOS curve should be identified with the OP's of the bands [3-5]. The present work carries this line of inquiry into the interesting and perhaps most relevant parameter range, namely, where the hopping strengths are comparable to the OP's or. $T_{c}$. For simplicity, we limit our work to two distinct layers in a unit cell with intralayer or interlayer pairing. It is found. that the results of both weak hopping and strong hopping theories are incomplete and potentially misleading. The features in DOS are not directly identifiable with the OP's of the layers, but are shifted and modified by the band dispersion along the $c$-direction. The singularities in DOS curves are logarithmic, which are less pronounced than those for BCS superconductors. We conclude that the tunneling curves for layered superconductors should be interpreted with caution.

The papers is organized as follows. Section 2 contains the analysis of a two-layer intralayer pairing model, showing the basic algebraic steps leading to our new conclusions.

Section 3 extends the consideration to the interlayer pairing case. Section 4 discusses how the results of the last two sections may be applied to real systems.

2. The Intralayer Pairing Model 
The model consists of two conducting layers in a unit cell with identical two-dimensional band structures and interlayer hopping strengths $J_{1}$ and $J_{2}$. The quasiparticles within the same layer interact via a phenomenological pairing interaction of the BCS type so that the total Hamiltonian has the form $H=H_{0}+V$, where the band energy term is

$$
\begin{aligned}
H_{0} & =\sum_{j \mathbf{k \sigma}} \sum_{n=1}^{2} \xi_{0}(\mathbf{k}) \psi_{j n \sigma}^{\dagger}(\mathbf{k}) \psi_{j n \sigma}(\mathbf{k}) \\
& +\sum_{j \mathbf{k} \sigma}\left[J_{1} \psi_{j 1 \sigma}^{\dagger}(\mathbf{k}) \psi_{j 2 \sigma}(\mathbf{k})+J_{2} \psi_{j 2 \sigma}^{\dagger}(\mathbf{k}) \psi_{j+1,1 \sigma}(\mathbf{k})+H . c .\right]
\end{aligned}
$$

where $\xi_{0}(\mathbf{k})=\mathrm{k}^{2} / 2 m_{0}-E_{F}, \mathbf{k}=\left(k_{x}, k_{y}\right), \sigma$ is the spin index, $n=1,2$ is the layer index within a unit cell, and the sum on $j$ is over all unit cells normal to the planes. The units are chosen such that $\hbar=k_{B}=1$. The interaction term is

$$
V=-\frac{1}{2} \sum_{j n} \sum_{\mathbf{k} \sigma} \sum_{\mathbf{k}^{\prime} \sigma^{\prime}} \lambda_{0 n} \psi_{j n \sigma}^{\dagger}(\mathbf{k}) \psi_{j n \sigma^{\prime}}^{\dagger}(-\mathbf{k}) \psi_{j n \sigma^{\prime}}\left(-\mathbf{k}^{\prime}\right) \psi_{j n \sigma}\left(\mathbf{k}^{\prime}\right)
$$

where we assume two unequal pair coupling strengths $\lambda_{0_{n}}$, both are cut off at a common energy $\omega_{\|}$. The condition $\sigma^{\prime}=-\sigma$ will be imposed, since the pair coupling mechanism only allows singlet pairs. The model has been generalized to two layers with different two-dimensional band structures and different Fermi surfaces [7], but the simpler model considered here gives a clearer physical picture.

The quasiparticle Green's function matrix elements are defined in the familiar way:

$$
\begin{aligned}
& G_{n n^{\prime}}\left(k, \tau-\tau^{\prime}\right)=-\left\langle T\left[\psi_{n \sigma}(k, \tau) \psi_{n^{\prime} \sigma}^{\dagger}\left(k, \tau^{\prime}\right)\right]\right\rangle, \\
& F_{n n^{\prime}}\left(k, \tau-\tau^{\prime}\right)=\left\langle T\left[\psi_{n \sigma}(k, \tau) \psi_{n^{\prime},-\sigma}\left(-k, \tau^{\prime}\right)\right]\right\rangle,
\end{aligned}
$$

where $k=\left(\mathrm{k}, k_{z}\right), k_{z}$ is the crystal momentum in the $c$-direction, $\langle\cdots\rangle$ denotes thermal average, and

$$
\psi_{n \sigma}(k)=\Omega^{1 / 2} \sum_{k_{z}} \psi_{j n \sigma}(\mathbf{k}) e^{i k_{z}[j s+(n-1) d]}
$$


In the above equation $\Omega$ is the volume of the crystal, $s$ is the thickness of the unit cell in the $c$-direction, and $d$ is the separation between layer 1 and layer 2 within the same cell. The separation between adjacent layers in adjacent cells will be denoted by $d^{\prime}=s-d$. The spin index is suppressed in the Green's function matrix elements. We define two order parameters $\Delta_{n}$, for $n=1,2$, by

$$
\Delta_{n}=\lambda_{0 n} \sum_{k^{\prime}}\left(\psi_{n \sigma}\left(k^{\prime}\right) \psi_{n,-\sigma}\left(-k^{\prime}\right)\right\rangle
$$

The inverse of the Green's function matrix has the form

$$
\hat{G}^{-1}(k, \nu)=\left(\begin{array}{cccc}
i \nu-\xi_{0}(\mathbf{k}) & -g\left(k_{z}\right) & \Delta_{1} & 0 \\
-g^{*}\left(k_{z}\right) & i \nu-\xi_{0}(\mathbf{k}) & 0 & \Delta_{2} \\
\Delta_{1}^{*} & 0 & i \nu+\xi_{0}(\mathbf{k}) & g\left(k_{z}\right) \\
0 & \Delta_{2}^{*} & g^{*}\left(k_{z}\right) & i \nu+\xi_{0}(\mathbf{k})
\end{array}\right),
$$

where the quantity

$$
g\left(k_{z}\right)=J_{1} e^{i k_{z} d}+J_{2} e^{-i k_{z} d^{\prime}} \equiv \epsilon_{\perp}\left(k_{z}\right) e^{-i \phi\left(k_{x}\right)}
$$

with

$$
\begin{aligned}
\epsilon_{\perp}\left(k_{z}\right) & =\left[J_{1}^{2}+J_{2}^{2}+2 J_{1} J_{2} \cos k_{z} s\right]^{1 / 2} \\
\phi\left(k_{z}\right) & =\frac{J_{2} \sin k_{z} d^{\prime}-J_{1} \sin k_{z} d}{J_{2} \cos k_{z} d^{\prime}+J_{1} \cos k_{z} d} .
\end{aligned}
$$

The functions $\pm \epsilon_{\perp}\left(k_{z}\right)$ represent the dispersion of the two tight-binding bands in the $c$ direction.

The inversion of the Green's function matrix is tedious but straightforward. Putting the results in Eq.(3), we deduce the following gap equations:

$$
\Delta_{1}=\frac{\lambda_{01}}{\beta} \sum_{k \nu} \frac{1}{D}\left\{\left[\nu^{2}+\xi_{0}^{2}(\mathbf{k})\right] \Delta_{1}+\epsilon_{\perp}^{2}\left(k_{z}\right) \Delta_{2}+\left|\Delta_{2}\right|^{2} \Delta_{1}\right\}
$$

where $\beta=1 / T, T$ is the temperature, and the sum over $\nu$ is over the Matsubara frequencies under the constraint that $|\nu|<\omega_{\|}$. The equation for $\Delta_{2}$ is similar except for an exchange 
of indices 1 and 2. The quantity $D$ is the determinant of the inverse Green's function matrix:

$D=\left(\nu^{2}+\xi_{0}^{2}\right)^{2}+2 \epsilon_{\perp}^{2}\left(\nu^{2}-\xi_{0}^{2}\right)+\epsilon_{\perp}^{4}+\left(\left|\Delta_{1}\right|^{2}+\left|\Delta_{2}\right|^{2}\right)\left(\nu^{2}+\xi_{0}^{2}\right)+\epsilon_{\perp}^{2}\left(\Delta_{1}^{*} \Delta_{2}+\Delta_{1} \Delta_{2}^{*}\right)+\left|\Delta_{1} \Delta_{2}\right|^{2}$.

As discussed in Ref. 3-5, in the state with minimum free energy the two order parameters are in phase.

The first step toward reducing the gap equations is to integrate over the two-dimensional crystal momentum $\mathbf{k}$. The results appear more compact in terms of the following set of new variables and parameters;

$$
\Delta_{ \pm}=\frac{1}{2}\left(\Delta_{1} \pm \Delta_{2}\right)
$$

and

$$
\lambda_{ \pm}=\frac{1}{2}\left(\lambda_{01} \pm \lambda_{02}\right)
$$

We find

$$
\begin{aligned}
\Delta_{ \pm}=\frac{\pi}{\beta} \sum_{\nu} \int \frac{s d k_{z}}{2 \pi} \frac{1}{2 c}\{ & \lambda_{ \pm} N(0)\left[\frac{c+\Delta_{-}^{2}-\epsilon_{\perp}}{\sqrt{\nu^{2}+a_{+}}}+\frac{c-\Delta_{-}^{2}+\epsilon_{\perp}}{\sqrt{\nu^{2}+a_{-}}}\right] \Delta_{+} \\
& \left.+\lambda_{\mp} N(0)\left[\frac{c+\Delta_{+}^{2}}{\sqrt{\nu^{2}+a_{+}}}+\frac{c-\Delta_{+}^{2}}{\sqrt{\nu^{2}+a_{-}}}\right] \Delta_{-}\right\}
\end{aligned}
$$

where $N(0)=m_{0} / 2 \pi s$ is the density of states per band,

$$
c=\left[\Delta_{+}^{2} \Delta_{-}^{2}-\epsilon_{\perp}\left(\Delta_{+}^{2}+\nu^{2}\right)\right]^{1 / 2}
$$

and

$$
a_{ \pm}=\Delta_{+}^{2}+\Delta_{-}^{2}-\epsilon_{\perp}^{2} \pm 2 c
$$

In the last step the sum over Matsubara frequencies is analytically continued into an integral over real frequencies $\omega$. The resulting integral equations are solved numerically for $\Delta_{n}(T)$. 
The critical temperature $T_{\mathrm{c}}$ is solved from the linearized version of Eq.(14). A brief summary of the results, which are discussed in detail in Ref. 6 and 8 , is as follows. In the limit of zero hopping the critical temperature is solved from

$$
\lambda_{>} N(0) a_{\|}\left(T_{c 0}\right)=1
$$

where $\lambda_{>}$is the larger of the two $\lambda_{0 n}$ and $a_{\|}(T)=\ln \left(2 \gamma \omega_{\|} / \pi T\right), \gamma=1.78$. With increasing hopping $T_{c}$ is lowered steadily from the zero hopping limit $T_{c 0}$. In the limit of strong hopping, i.e. $J_{n} \gg \omega_{\|}$, the critical temperature is solved from

$$
\lambda_{+} N(0) a_{\|}\left(T_{c}\right)=1
$$

with $\lambda_{+}$defined in Eq.(12).

The OP's at zero temperature depend also on the hopping strengths. In the zero hopping limit the equations separate into two independent equations whose solutions are the gaps for the individual layers. With increasing hopping the larger of the two solutions, which is designated as $\Delta_{1}$, decreases while the smaller one, $\Delta_{2}$ increases. Thus, the two layers are more similar to each other in their superconducting properties."- In the strong hopping limit, we find that the ratio of the gaps satisfies $\Delta_{1} / \Delta_{2}=\lambda_{1} / \lambda_{2}$. The average gap $\Delta_{+}$defined in Eq.(12) is the energy gap of the energy bands. These results are summarized in Fig.1 for a set of parameters $\lambda_{02} / \lambda_{01}=0.7, \lambda_{01} N(0)=0.5, J_{2} / J_{1}=0.3$, and $J_{1} / T_{c 0}$ ranging from 0.1 to 10 .

In Fig.2 we show $\Delta_{n}(T), n=1,2$, for the same of model parameters except that $J_{1} / T_{c 0}=1$. For these parameters the critical temperature $T_{c}=0.92 T_{c 0}$. The dominant OP $\Delta_{1}$ has the typical BCS behavior. The lesser OP $\Delta_{2}$ initially behaves as if it is heading toward a critical temperature lower than $T_{c}$, but turns around to follow $\Delta_{1}$ above $0.6 T_{c}$, where superconductivity in layer 2 is induced by the proximity effect. This result is typical for systems with two or more coupled order parameters. 
The density-of-states in the superconducting state is calculated in the standard way:

$$
N_{s}(\omega)=\left.\frac{1}{\pi} \sum_{k \mathbf{n}} \operatorname{ImG}_{\mathrm{nn}}(\mathrm{k}, \nu)\right|_{\nu \rightarrow-\mathrm{i} \omega+\delta}
$$

where $\delta=0^{+}$. After integrating over $\mathbf{k}$, we find

$$
N_{s}(\omega)=N(0) \int \frac{s d k_{z}}{2 \pi} \operatorname{Im} \frac{-\mathrm{i} \nu}{2 \mathrm{c}}\left[\frac{\mathrm{c}-\epsilon_{\perp}^{2}}{\sqrt{\nu^{2}+\mathrm{a}_{+}}}+\frac{\mathrm{c}+\epsilon_{\perp}^{2}}{\sqrt{\nu^{2}+\mathrm{a}_{-}}}\right]_{\nu \rightarrow-\mathrm{i} \omega+\delta}
$$

where the quantities $c$ and $a_{ \pm}$are defined in Eqs.(14) and (15). We show in Fig.3 a set of total DOS curves at zero temperature for the same set of model parameters in Fig.1 with $J_{1} / T_{c 0}$ ranging from a weak hopping value of 0.1 to a strong hopping value of 5 . The calculated $\Delta_{1,2}$ are marked on the graph with two kinds of arrows. The four curves are displaced vertically by 2 units successively. It can be seen that in the weak hopping case; the bottommost curve, the DOS has two sets of peaks corresponding to the OP's of the two layers, in agreement with the conclusions of Refs. 1 and 2. Each set resembles the BCS result. The system may be regarded as two nearly independent superconductors, each with its own energy gap. The strong hopping case, the topmost curve, the peaks are situated halfway between $\Delta_{1}$ and $\Delta_{2}$, i.e. the position of $\Delta_{+}$, in agreement with Refs. 3-5. The two middle curves for intermediate hopping do not conform to either physical picture. In particular, for $J_{1} / T_{c 0}=1$, we find $N_{s}(\omega)=0$ for $\omega<\omega_{0}^{\prime \prime}=\Delta_{+} \sqrt{1-\Delta_{-}^{2} /\left(J_{1}-J_{2}\right)^{2}}$. [In calculating the curves in Fig. 3 we have put in a nonvanishing $\delta=0.3-0.5$ in Eq.(20) to smooth out the curves, and this results in a small and nonvanishing DOS inside of the threshold]. The first peak occurs at $\omega_{0}^{\prime}=\Delta_{+} \sqrt{1-\Delta_{-}^{2} /\left(J_{1}+J_{2}\right)^{2}}$. In the limit of $\delta=0$, the peak is a logarithmic singularity, i.e.

$$
N_{s}(\omega)=A_{ \pm} \ln \left|\omega-\omega_{0}^{t}\right|+B_{ \pm}
$$

where $A_{ \pm}, B_{ \pm}$are nonsingular in $\omega$ and \pm refer to the high or low side of the singularity. A second logarithmic singularity occurs at $\omega=\omega_{+}^{\prime}=\sqrt{\left(J_{1}+J_{2}\right)^{2}+\Delta_{+}^{2}}+\Delta_{-}$, which is 
outside of $\omega_{0}^{\prime}$. Both singularies are less sharp than the $(\omega-\Delta)^{-1}$ singularity of a BCS superconductor, and a dip in the DOS curve occurs between them. The detail of this analysis will be given in the Appendix. It should be noted that the two sets of peaks only occur when $\lambda_{01} \neq \lambda_{02}$. For equal coupling strengths we find that $\Delta_{1}=\Delta_{2}$ and the DOS follows the standard BCS behavior [3-5].

The tunneling characteristics at various temperatures are calculated by convoluting the DOS curve with the Fermi distribution factor:

$$
\frac{d I(V)}{d V} \propto \int N_{s}(\omega-e V) \frac{\beta e^{\beta \omega}}{\left(e^{\beta \omega}+1\right)^{2}} d \omega
$$

where $e$ is quasiparticle charge and $V$ is the bias. We show in. Fig.4 a set of tunneling . characteristics for the same set of material parameters used in Fig.2. The temperatures. are marked on the curves. At elevated temperatures the fine details of the DOS curves tend to be smoothed away, with only a shallow dip left around zero bias. These:curves resemble qualitatively the $a b$-direction tunneling data of BSSCO $[9,10]$.

High- $\mathrm{T}_{c}$ materials all have short coherence lengths in the $c$-direction. Consequently, when tunneling in this direction, the data may be more representative of the topmost layer then the bulk. We simulate this effect by projecting out the DOS of the individual layers:

$$
N_{s}^{(n)}(\omega)=N(0) \int \frac{s d k_{z}}{2 \pi} \operatorname{Im} \frac{-\mathrm{i} \nu}{2 \mathrm{c}}\left[\frac{-\epsilon_{\perp}^{2}+\mathrm{c} \pm \Delta_{+} \Delta_{-}}{\sqrt{\nu^{2}+\mathrm{a}_{+}}}+\frac{\epsilon_{\perp}^{2}+\mathrm{c} \mp \Delta_{+} \Delta_{-}}{\sqrt{\nu^{2}+\mathrm{a}_{-}}}\right]_{\nu \rightarrow-\mathrm{i} \omega+\delta}
$$

where the upper (lower) sign applies to $n=1(2)$. The tunneling characteristics based on the projected DOS are shown in Figs. 5 and 6 . These curves are visibly different from those in Fig.4, especially the relative weight of the two peaks. Thus, depending which layer is exposed, the c-axis tunneling data may differ considerably from sample to sample and from the $a b$-axes data. In the next section we extend these considerations to the interlayer pairing model. 
3. The Interlayer Pairing Model

The interlayer pairing model postulates that the Cooper pairs always reside in adjacent layers. The interaction Hamiltonian $V$ which leads to this type of pairs is of the form

$$
\begin{aligned}
V= & -\sum_{j} \sum_{\mathbf{k \sigma}} \sum_{\mathbf{k}^{\prime} \sigma^{\prime}}\left[\lambda_{1} \psi_{j 1 \sigma^{\prime}}^{\dagger}(\mathbf{k}) \psi_{j 2 \sigma^{\prime}}^{\dagger}(-\mathbf{k}) \psi_{j 2 \sigma^{\prime}}\left(-\mathbf{k}^{\prime}\right) \psi_{j 1 \sigma}\left(\mathbf{k}^{\prime}\right)\right. \\
& \left.+\lambda_{2} \psi_{j 2 \sigma}^{\dagger}(\mathbf{k}) \psi_{j+1,1 \sigma^{\prime}}^{\dagger}(-\mathbf{k}) \psi_{j+1,1 \sigma^{\prime}}\left(-\mathbf{k}^{\prime}\right) \psi_{j 2 \sigma}\left(\mathbf{k}^{\prime}\right)\right]
\end{aligned}
$$

where $\lambda_{1,2}$ are the intracell and intercell coupling strengths, both are cut off at a common energy $\omega_{\perp}$. It has been shown in Refs $3-5$ that the interlayer pairing interaction favors singlet pairing, although in a certain range of parameter space the triplet pairing may emerge at a temperature below $T_{c}$. We will concentrate in this paper the large region of the parameter space in which the triplet state can be ignored altogether.

The quasiparticle Green's function matrix elements are defined the same way as in Eq.(3). We define two order parameters

$$
\begin{aligned}
& \Delta_{1}=\lambda_{1} \sum_{\mathbf{k}^{\prime}}\left\langle\psi_{j 1 \sigma}\left(\mathbf{k}^{\prime}\right) \psi_{j 2,-\sigma}\left(-\mathbf{k}^{\prime}\right)\right\rangle \\
& \Delta_{2}=\lambda_{2} \sum_{\mathbf{k}^{\prime}}\left\langle\psi_{j 2,-\sigma}\left(-\mathbf{k}^{\prime}\right) \psi_{j+1,1 \sigma}\left(\mathbf{k}^{\prime}\right)\right\rangle .
\end{aligned}
$$

We can then write the inverse Green's function matrix as

$$
G^{-1}(k, \nu)=\left(\begin{array}{cccc}
i \nu-\xi_{0}(\mathbf{k}) & -g\left(k_{z}\right) & 0 & -\Delta_{a} e^{-i \phi\left(k_{z}\right)} \\
-g^{*}\left(k_{z}\right) & i \nu-\xi_{0}(\mathbf{k}) & -\Delta_{b} e^{i \phi\left(k_{z}\right)} & 0 \\
0 & -\Delta_{b}^{*} e^{-i \phi\left(k_{z}\right)} & i \nu+\xi_{0}(\mathbf{k}) & g\left(k_{z}\right) \\
-\Delta_{a}^{*} e^{i \phi\left(k_{z}\right)} & 0 & g^{*}\left(k_{z}\right) & i \nu+\xi_{0}(\mathbf{k})
\end{array}\right)
$$

with

$$
\begin{aligned}
& \Delta_{a}=\Delta_{1} e^{i\left[k_{z} d+\phi\left(k_{z}\right)\right]}+\Delta_{2} e^{-i\left[k_{z} d^{\prime}-\phi\left(k_{z}\right)\right]}, \\
& \Delta_{6}=\Delta_{1} e^{-i\left[k_{z} d+\phi\left(k_{z}\right)\right]}+\Delta_{2} e^{i\left[k_{z} d^{\prime}-\phi\left(k_{z}\right)\right]} .
\end{aligned}
$$


The physical meaning of $\Delta_{a}$, and $\Delta_{b}$ will be discussed later. In the ground state where $\Delta_{1}$ and $\Delta_{2}$ are in phase and real, we have $\Delta_{b}=\Delta_{a}^{*}$. The gap equations as derived in Ref. 8 are:

$$
\begin{aligned}
& \Delta_{1}=\frac{\lambda_{1}}{\beta} \sum_{k \nu} \frac{1}{D}\left\{\left[\nu^{2}+\xi_{0}^{2}(\mathbf{k})\right] \Delta_{a}+\epsilon_{\perp}^{2}\left(k_{z}\right) \Delta_{a}^{*}+\left|\Delta_{a}\right|^{2} \Delta_{a}\right\} e^{-i\left[k_{z} d+\phi\left(k_{z}\right)\right]} \\
& \Delta_{2}=\frac{\lambda_{2}}{\beta} \sum_{k \nu} \frac{1}{D}\left\{\left[\nu^{2}+\xi_{0}^{2}(\mathbf{k})\right] \Delta_{a}+\epsilon_{\perp}^{2}\left(k_{z}\right) \Delta_{a}^{*}+\left|\Delta_{a}\right|^{2} \Delta_{a}\right\} e^{i\left[k_{z} d^{\prime}-\phi\left(k_{z}\right)\right]}
\end{aligned}
$$

The sums over $\nu$ are cut off at $\omega_{\perp}$. The quantity $D$ has the expression

$$
D=\left(\nu^{2}+\xi_{0}^{2}\right)^{2}+2 \epsilon_{\perp}^{2}\left(\nu^{2}-\xi_{0}^{2}\right)+\epsilon_{\perp}^{4}+2\left|\Delta_{a}\right|^{2}\left(\nu^{2}+\xi_{0}^{2}\right)+\epsilon_{\perp}^{2}\left(\Delta_{a}^{2}+\Delta_{a}^{* 2}\right)+\left|\Delta_{a}\right|^{4}:
$$

After integrating over the two-dimensional crystal momentum $\mathbf{k}$, we obtain

$$
\begin{aligned}
& \Delta_{1}=\lambda_{1} N(0) \frac{\pi}{\beta} \sum_{\nu} \int \frac{s d k_{z}}{2 \pi} I\left(k_{z}\right) e^{-i\left[k_{z} d+\phi\left(k_{z}\right)\right]}, \\
& \Delta_{2}=\lambda_{2} N(0) \frac{\pi}{\beta} \sum_{\nu} \int \frac{s d k_{z}}{2 \pi} I\left(k_{z}\right) e^{i\left[k_{z} d^{*}-\phi\left(k_{x}\right)\right]}
\end{aligned}
$$

where the integrand

$$
I\left(k_{z}\right)=\frac{1}{2}\left\{\left[\Delta_{a}+\frac{i \epsilon_{\perp}\left(k_{z}\right)}{c}\right] \frac{1}{\sqrt{\nu^{2}+a_{+}}}+\left[\Delta_{a}-\frac{i \epsilon_{\perp}\left(k_{z}\right)}{c}\right] \frac{1}{\sqrt{\nu^{2}+a_{-}}}\right\} .
$$

These equations have structures similar to the intralayer pairing case, but the various quantities therein have different definitions:

$$
c=\left[\nu^{2}+\left(\operatorname{Re} \Delta_{a}\right)^{2}\right]^{1 / 2}
$$

and

$$
a_{ \pm}=\left|\Delta_{a}\right|^{2}-\epsilon_{\perp}^{2} \pm 2 i \epsilon_{\perp} c
$$

We review briefly here the behavior of the the critical temperature as a function of hopping as discussed in detail in Refs.6 and 8. In the limit of zero hopping the critical temperature is determined by the stronger of the two coupling constants via the equation

$$
\lambda_{>} N(0) a_{\perp}\left(T_{c 0}\right)=1
$$


where, in analogy with Eq.(18), $a_{\perp}(T)=\ln \left(2 \gamma \omega_{\perp} / \pi T\right)$. With increasing hopping, the critical temperature $T_{c}$ diminishes steadily from $T_{c 0}$. In the strong hopping limit, the asymptotic $T_{c}$ is solved from

$$
\left[N(0) a_{\perp}\left(T_{c}\right)\right]^{-1}=\frac{1}{4}\left\{\lambda_{1}+\left(2-\zeta^{2}\right) \lambda_{2}+\left[\lambda_{1}^{2}-2\left(2-3 \zeta^{2}\right) \lambda_{1} \lambda_{2}+\left(2-\zeta^{2}\right)^{2} \lambda_{2}^{2}\right]^{1 / 2}\right\}
$$

with $\zeta=J_{1} / J_{2}$. At zero temperature and zero hopping, only $\Delta_{1}$, which corresponds to the larger coupling strength $\lambda_{1}$, is nonvanishing. For stronger hopping $\Delta_{1}$ diminishes while $\Delta_{2}$ grows, similar to the intralayer pairing case shown in Fig.1.

In Fig. 7 we show the results of the numerical solutions of the finite temperature gap equations, Eqs.(33) and (34), for a set of parameters $\lambda_{2} / \lambda_{1}=0.5, \lambda_{1} N(0)=0.5, J_{2} / J_{1}=$ 0.8 , and $J_{1} / T_{c 0}=1$. For these parameters $T_{c}=0.95 T_{c 0}$. The dominant OP $\Delta_{1}$ has the BCS temperature dependence, while the subordinate OP $\Delta_{2}$ is small over the entire temperature range. This is the general behavior for weak to intermediately hopping, that one OP dominates over the other nearly completely. The pattern changes when hopping is increased, as shown in Fig. 8 for a set of parameters $\lambda_{2} / \lambda_{1}=0.8, \lambda_{1} N(0)=0.5, J_{1} / J_{2}=0.4$, and $J_{2} / T_{c 0}=2$. It can be seen that $\Delta_{2}$ is the stronger OP at low temperatures but loses its dominance at high temperatures. This type of competition between OP's, not seen in the intralayer pairing case, arises from the fact that the OP's are nonlocal and interact more effectively through the $c$-axis band dispersion than the local OP's of intralayer pairing. The nonlocal nature of the OP's also manifests itself in additional structures in the DOS and tunneling characteristics to be discussed next.

The expression for the DOS in the superconducting state as calculated from Eq.(19) is found to be, after integrating over $\mathrm{k}$ :

$$
\begin{aligned}
N_{s}(0) & =N(0) \int \frac{s d k_{z}}{2 \pi} \operatorname{Im}(-i \nu)\left\{\left[1+\frac{i \epsilon_{\perp}}{\sqrt{\nu^{2}+\left(\operatorname{Re} \Delta_{a}\right)^{2}}}\right] \frac{1}{\sqrt{\nu^{2}+a_{+}}}\right. \\
& \left.+\left[1-\frac{i \epsilon_{\perp}}{\sqrt{\nu^{2}+\left(\operatorname{Re} \Delta_{a}\right)^{2}}}\right] \frac{1}{\sqrt{\nu^{2}+a_{-}}}\right\}_{\nu \rightarrow-i \omega+\delta}
\end{aligned}
$$


A set of DOS curves is shown in Fig.9 for model parameters used in Fig.7 except that $J_{1} / T_{c 0}$ ranges from 0.1 to 5 . The bottommost curve for weak hopping is BCS-like, with peaks at $\pm \Delta_{1}$, i.e. the dominant OP. The topmost curve for strong hopping has threshold at $\pm\left(\Delta_{1}-\Delta_{2}\right)$ and main peaks at $\pm\left(\Delta_{1}+\Delta_{2}\right)$. The DOS curve increases continuously between the threshold and the peak on both sides. The origin of these features has been discussed in Ref.3-5, and is reiterated here using a different language. Recall the definition of $\Delta_{a}$ and its complex conjugate $\Delta_{b}$ in Eqs.(28) and (29). The real part of $\Delta_{a}$ or $\Delta_{b}$ given by:

$$
\operatorname{Re} \Delta_{a}=\Delta_{1} \cos \left[k_{z} d+\phi\left(k_{z}\right)\right]+\Delta_{2} \cos \left[k_{z} d^{\prime}-\phi\left(k_{z}\right)\right]
$$

which is an even function of $k_{z}$, is the intraband OP discussed in early papers, ${ }^{3-5}$. while the imaginary part, an odd function of $k_{z}$ given by:

$$
\operatorname{Im} \Delta_{a}=\Delta_{1} \sin \left[k_{z} d+\phi\left(k_{z}\right)\right]-\Delta_{2} \sin \left[k_{z} d^{\prime}-\phi\left(k_{z}\right)\right]
$$

is the interband OP. It can be shown without much difficulty that the imaginary part van-. ishes in the strong hopping limit; leaving the intraband OP as the only order parameter. A little algebra yields that the extremal values of the right-hand-side of $\mathrm{Eq}(39)$ are $\Delta_{1} \pm \Delta_{2}$ obtained at $k_{z}=0$ and $k_{z}= \pm \pi / s$ respectively. Therefore, the inner structure reflects the $c$-axis versus $a b$-plane anisotropy of the gap function [3-5]. The main peaks are logarithmic singularities because of the $k_{z}$ dependence of the gap function. In the intermediate hopping regime the DOS curves exhibit two sets of peaks in addition to structures inside of the inner peaks. It will be discussed in the Appendix that both sets of peaks are logarithmic singularities, but it is not straightforward to write down analytic expressions for the threshold and the positions of the singularities. Nonetheless, one can establish that, just like the intralayer pairing case, the outer peaks are associated with interband pairing. In contrast to the intralayer problem, however, the interband peaks may be stronger than 
the intraband peaks, as demonstrated in Fig.10. The interpretation of the DOS curve is further complicated by the fact that both band dispersion and gap function dispersion contribute to the shape of the inner structure.

A set of simulated tunneling characteristics is shown in Fig.11. The general behavior is similar to the intralayer pairing case, that all features are smeared by thermal broadening. The curves bear close resemblance to the actual data on YBCO $[10,11]$.

\section{Summary and Conclusion}

We have demonstrated that, simply as a result of the layered structure, the copperoxide superconductors may have multiple peaks in their density-of-states and tunneling characteristics. Careful analyses of high quality tunneling data may shed light on the pairing mechanism. Because the short $c$-axis coherence lengths, the preferred way to take data is by tunneling out of the $a$ and $b$-planes.

Our theoretical results, though limited in scope, may be applicable to real materials. Consider the case of $\mathrm{YBCO}$ with two identical $\mathrm{CuO}$ layers. If these are the only superconducting layers and the pairing mechanism is intralayer, we would conclude that the DOS should be BSC-like, in disagreement with the experiments $[10,11]$. We speculate, therefore, that either the $\mathrm{CuO}$ chains form another superconducting layer, or that the pairing mechanism is interlayer. One may be able to distinguish between the two possibilities by a detailed examination of the inner structure of the $a b$-plane tunneling data. The BSSCO system is inherently more complex owing to the additional $\mathrm{BaO}$ layers, which may be superconducting. Without solving the more than two layer model, we anticipate that the main DOS peaks are due to intraband pairing and should be logarithmic singularities. On the other hand, there should be more than one interband pairing features each associated with a different pair of bands, resulting in a less clear outer peak. Only the dip outside of the main peak remains as indication of the multilayer nature of the material. Again, any 
additional structure inside the main peaks may hint at interlayer pairing. On the other hand, the absence of inner feature does not necessarily rule out interlayer pairing for the following reason. It has been shown in Ref. 8 that if the hopping strength between two layers is stronger than the OP's, the two layers would act like one layer with intralayer pairing even though the pairing between the two layers in interlayer. In view of these complications, we would urge extra care in interpreting the tunneling data of high- $T_{c}$ superconductors. It is interesting to note that the layered organic superconductor $\kappa$-(BEDT-TTF $)_{2} \mathrm{Cu}(\mathrm{NCS})_{2}$ displays the same double peak tunneling characteristic although its critical temperature is merely $11 \mathrm{~K} \mathrm{[12].} \mathrm{We} \mathrm{view} \mathrm{this} \mathrm{as} \mathrm{added} \mathrm{evidence} \mathrm{that} \mathrm{the} \mathrm{crystal} \mathrm{geometry,} \mathrm{rather} \mathrm{than}$ the coupling mechanism, is the cause of the observed complex gap structure.

5. Acknowledgments

This work was supported by the U.S. Department of Energy, Division of Basic Energy Sciences, under contracts no. DE-AC005-84OR21400 with Martin Marietta Energy Systems, Inc., and no. W-31-109-ENG-38. 
References

[1] M. Tachiki, S. Takahashi, F. Steglich and H. Adrian, Z. Phys. B 80 (1990) 161.

[2] L. N. Bulaevskii and M. V. Zyskin, Phys. Rev. B 41 (1990) 873.

[3] R. A. Klemm and S. H. Liu, Physica C 176 (1991) 189 .

[4] R. A. Klemm and S. H. Liu, Phys. Rev. B 44 (1991) 7526.

[5] S. H. Liu and R. A. Klemm, Phys. Rev. B 45 (1992) 415.

[6] R. A. Klemm and S. H. Liu, Physica C 191 (1992) 383.

[7] A. A. Abrikosov and R. A. Klemm, Physica C 191 (1992) 224.

[8] S. H. Liu and R. A. Klemm, Phys. Rev. B (to appear).

[9] Q. Chen and K.-W. Ng, Phys. Rev. B 45 (1992) 2569.

[10] T. Hasegawa, M. Nantoh, A. Takagi, H. Ikuta, M. Kawasaki, H. Koinuma and K: Kitawawa, J. Phys. Chem. Solid 53 (1992) 1643.

[11] M Gurvitch, J. M. Valles, Jr., A. M. Cucolo, R. C. Dynes, J. P. Garno, L. F. Schneemeyer and J. V. Waszczak, Phys. Rev. Lett. 63 (1989) 329.

[12] H. Bando, S. Kashiwaya, H. Tokumoto, H. Anzai, N. Kinoshita and K. Kajimura, J. Vac. Sci. Technol. A 8 (1990) 479. 
Figure Captions

1. The dependence of the layer order parameters on the hopping strengths for a two-layer model with intralayer pairing. The model parameters are displayed in the graph.

2. The temperature dependence of the layer order parameters for a two-layer model with intralayer pairing. The model parameters are displayed in the graph.

3. A set of density-of-states (DOS) curves at zero temperature for a two-layer model with intralayer pairing. The model parameters are given in Fig.1. The curves are displaced vertically by 2 units successively.

4. Simulated tunneling characteristics for the two-layer model whose model parameters are in Fig.2. The curves are displaced vertically by 2 units successively.

5. Simulated tunneling characteristics from the layer 1 of the two-layer model studied in Figs.2 and 3. The curves are displaced vertically by 2 units successively.

6. Simulated tunneling characteristics from the layer 2 of the two-layer model studied in Figs.2 and 3. The curves are displaced vertically by 2 units successively.

7. The temperature dependence of the interlayer order parameters for a two-layer model with interlayer pairing. The model parameters are displayed in the graph.

8. The temperature dependence of the interlayer order parameters for a two-layer with a different set of model parameters. The model parameters are displayed in the graph. The OP $\Delta_{2}$ dominates at low temperatures but becomes less than $\Delta_{1}$ near $T_{c}$.

9. A set of DOS curves at zero temperature for a two-layer model with interlayer pairing. The model parameters are such that $\lambda_{2} / \lambda_{1}=0.5, \lambda_{1} N(0)=0.5, J_{1} / J_{2}=0.8$, and $J_{1} / T_{c 0}$ ranging from 0.1 to 5 . The curves are displayed vertically by 2 units successively.

10. A set of DOS curves at zero temperature for a two-layer model with interlayer pairing. The model parameters are such that $\lambda_{2} / \lambda_{1}=0.8, \lambda_{1} N(0)=0.5, J_{1} / J_{2}=0.4$, 
and $J_{1} / T_{c 0}$ ranging from 0.1 to 5 . The curves are displayed vertically by 2 units successively.

11. Simulated tunneling characteristics for a two-layer model with interlayer pairing. The model parameters are given in Fig.9. 


\section{Appendix}

We analyze here the structure of the DOS curves for the two-layer model with intralayer or interlayer pairing. The expression for $N_{0}(\omega)$ for the intralayer model is given in Eq.(20). After making the analytical continuation to real frequencies, we find that the right-hand side of Eq.(20) have singularities determined by the zeros of the factors $a_{+}-\omega^{2}, a_{-}-\omega^{2}$, and $c$, where

$$
c=\sqrt{\Delta_{+}^{2} \Delta_{-}^{2}+\epsilon_{\perp}^{2}\left(\omega^{2}-\Delta_{+}^{2}\right)},
$$

and $a_{ \pm}$are given in Eq.(16). The factor $c$ has a zero at

$$
\omega_{0}=\Delta_{+} \sqrt{1-\frac{\Delta_{-}^{2}}{\epsilon_{\perp}^{2}}}
$$

which is real for $\epsilon_{\perp}>\Delta_{-}$. The quantity $\epsilon_{\perp}$, given in Eq.(8), is a function of of the c-axis momentum $k_{z}$. Consequently $\omega_{0}$ varies from its maximum $\omega_{0}^{\prime}=\Delta_{+} \sqrt{1-\Delta_{-}^{2} /\left(J_{1}+J_{2}\right)^{2}}$ obtained at $k_{z}=0$ to its minimum $\omega_{0}^{\prime \prime}=\Delta_{+} \sqrt{1-\Delta_{-}^{2} /\left(J_{1}-J_{2}\right)^{2}}$ obtained at $k_{z}= \pm \pi / s$. The zeros of $a_{ \pm}-\dot{\omega}^{2}$ are more subtle. One can show after some manipulations that

$$
\left(a_{+}-\omega^{2}\right)\left(a_{-}-\omega^{2}\right)=\left(\omega_{+}^{2}-\omega^{2}\right)\left(\omega_{-}^{2}-\omega^{2}\right)
$$

where

$$
\omega_{ \pm}=\sqrt{\epsilon_{\perp}^{2}\left(k_{z}\right)+\Delta_{+}^{2}} \pm \Delta_{-}
$$

Thus the zeros of $a_{ \pm}-\omega^{2}$ are contained in the set $\pm \omega_{ \pm}$. Further analysis reveals that $\omega_{+}$is a zero of $a_{+}-\omega^{2}$ only. The quantity $\omega_{-}$, however, is a zero of $a_{+}-\omega^{2}$ or $a_{-}-\omega^{2}$. depending on whether $\epsilon_{\perp}^{2}$ is greater than or less than the following quantity:

$$
b=\Delta_{-} \sqrt{\epsilon_{\perp}^{2}+\Delta_{+}^{2}}
$$

Bear in mind that $\epsilon_{\perp}$ is a function of $k_{z}$, it follows that both $\omega_{ \pm}$vary between their maximum values $\omega_{ \pm}^{\prime}=\sqrt{\left(J_{1}+J_{2}\right)^{2}+\Delta_{+}^{2}} \pm \Delta_{-}$at $k_{z}=0$ and minimum values $\omega_{ \pm}^{\prime \prime}=$ 
$\sqrt{\left(J_{1}-J_{2}\right)^{2}+\Delta_{+}^{2}} \pm \Delta_{-}$at $k_{z}= \pm \pi / s$. The zero at $\omega_{-}$may shift from one term in the square bracket of Eq.(20) to the other depending on the value of $k_{z}$. The following inequality $\omega_{+} \geq \omega_{-} \geq \omega_{0}$ is satisfied for all values of $k_{z}$.

We will make use of this set of information to analyze the DOS curves. In the weak coupling limit the quantity $c$ is real for all values of $\omega$, and the quantities $\omega_{ \pm}=\Delta_{1}, \Delta_{2}$ respectively. Therefore, the singuarities of $N_{s}(\omega)$ are the order parameters of the individual layers. In the strong hopping limit the quantity $\omega_{0}=\Delta_{+}$and $\omega_{ \pm}$are so large that they are out of the picture. The only singularity in DOS corresponds to the energy gap in the band representation. For intermediate hopping one must study the singular behavior on individual basis. As an example, consider the case $J_{1} / T_{c 0}=1$ in Fig.4. The input data are, $\Delta_{1}=1.7416, \Delta_{2}=1.2927, J_{1}=1, J_{2}=0.3$, all in units of $T_{c 0}$. From these we determine at $k_{z}=0$ that $\omega_{+}^{\prime}=2.2823, \omega_{-}^{\prime}=1.8333$ and $\omega_{0}^{\prime}=1.2131$. The inequality $\epsilon_{\perp}^{2}>b$ is satisfied, $b$ is defined in Eq.(A5). At the zone boundary, $k_{z}= \pm \pi / s$, we find $\omega_{+}^{\prime \prime}=1.9191, \omega_{-}^{\prime \prime}=1.0211, \omega_{0}^{\prime \prime}=0.9917$, and $\epsilon_{\perp}^{2}<b$. Thus, the following picture emerges: For $\omega<\omega_{0}^{\prime \prime}$ the factor $c$ is real and $N_{s}(\omega)=0$ because the integrand in $\mathrm{Eq}(20)$ vanishes. In the numerical calculation a small but finite $\delta=.05$ is used to help smooth the curves, but this also causes the $N_{s}(\omega)$ curves to appear nonvanishing inside of the threshold. For $\omega$ just beyond the threshold, the DOS increases rapidly because a larger range of $k_{z}$ near the zone boundary now contributes. This trend continues until $\omega$ reaches $\omega_{0}^{\prime}$ where the entire zone of $k_{z}$ contributes to the integral in Eq.(20). It is not difficult to show that the $k_{z}$ integral actually diverges logarithmically, which implies the asymptotic property of $N_{s}(\omega)$ in Eq.(21). It should be noted that the main peak is contained in the factor $c$, as in the strong hopping case.

A similar reasoning applies to the second logarithmic singularity at $\omega_{+}^{\prime}$. This singularity is contained in the factor $a_{+}-\omega^{2}$, reminiscent of the weak hopping case except that 
the band splitting contained in the expression for $\omega_{+}$(Eq.(A4)) is not negligible. For this reason as well as that this feature disappears in the strong hopping limit, we identify it as the interband pairing contribution to $N_{s}(\omega)$. Because $\omega_{0}^{\prime}>\omega_{-}^{\prime \prime}$ the singularity at $\omega_{-}$is overwhelmed by the main peak.

The singularities for the interlayer pairing problem are investigated in the same manner. The expression for $N_{s}(\omega)$ is given in Eq.(39), with $a_{ \pm}$in Eq.(36). One singularity can be readily identified as $\omega_{0}=\left|\operatorname{Re} \Delta_{\mathrm{a}}\left(k_{\mathrm{z}}\right)\right|$, which is the intraband gap function. As in the intralayer pairing model, the $k_{z}$ dispersion of this singularity determines the threshold, the inner structure and the nature of the main peak in the strong hopping case. Additional singularities are found from the relation:

$$
\left(a_{+}-\omega^{2}\right)\left(a_{-}-\omega^{2}\right)=\left(\omega_{+}^{2}-\omega^{2}\right)\left(\omega_{-}^{2}-\omega^{2}\right),
$$

where

$$
\omega_{ \pm}^{2}=\left(\operatorname{Re} \Delta_{a}\right)^{2}+\left(\epsilon_{\perp} \pm \operatorname{Im} \Delta_{a}\right)^{2}
$$

The $k_{z}$ dependence of $\omega_{ \pm}$depends on both the gap function dispersion and the band dispersion, making the outer peak difficult to locate analytically: Numerically, we find that the outer peak is at the maximum value of $\omega_{+}$, which occurs in the middle of the $c$-axis Brillouin zone. 


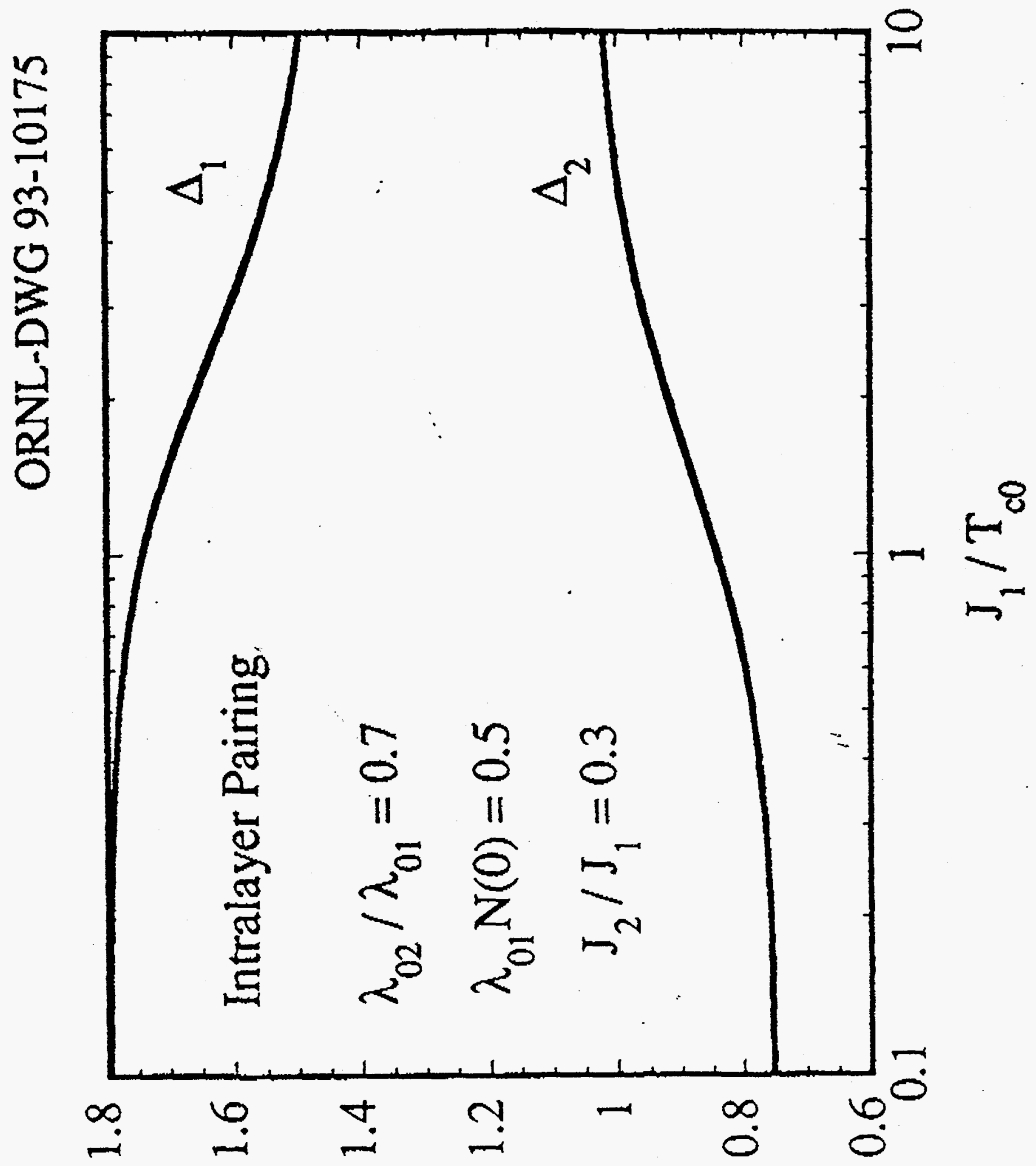

${ }^{00} \mathrm{~L} /{ }^{2} \mathrm{I} \nabla$

Fins 1 


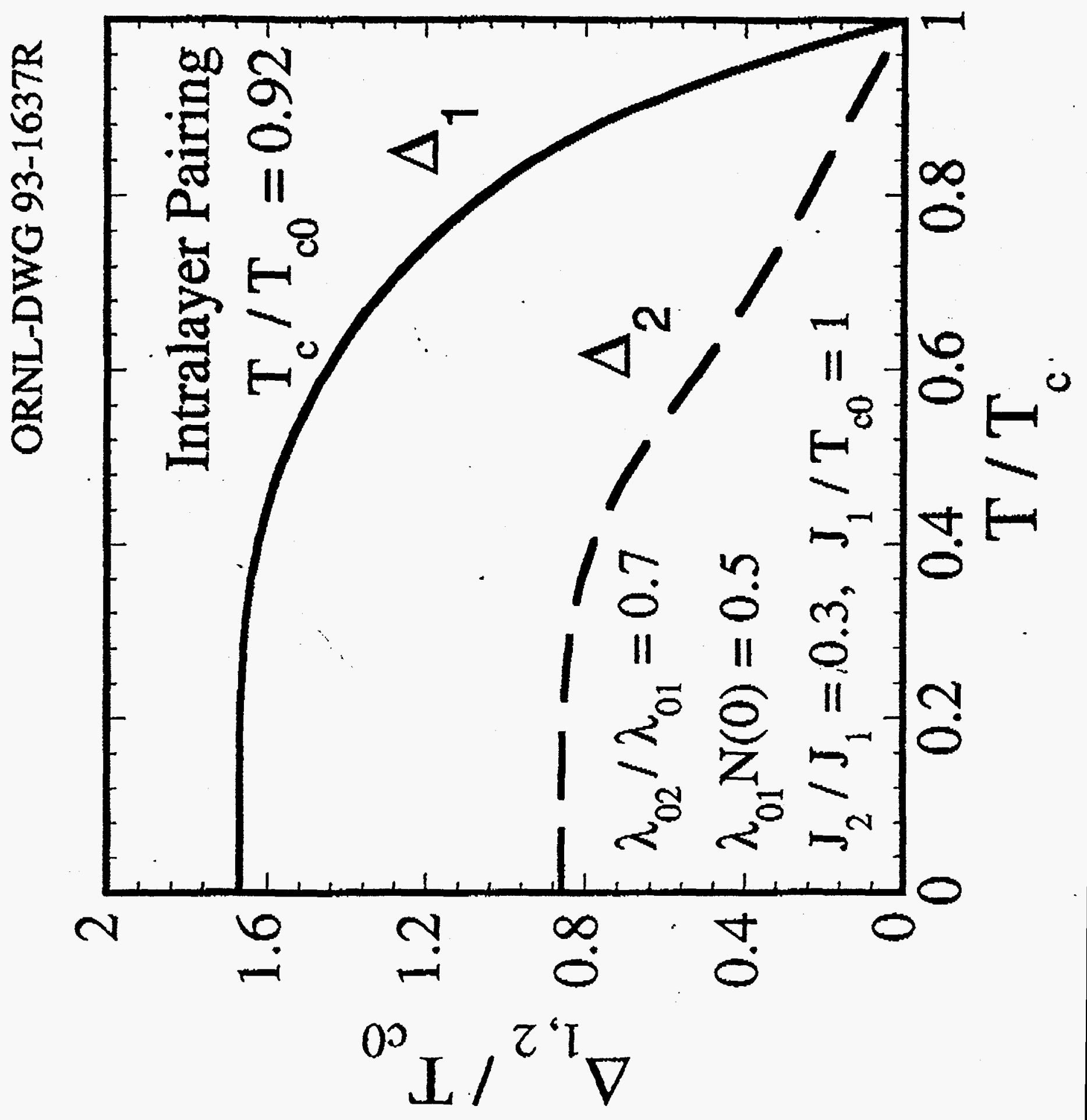

$F=2$ 
ORNL-DWG 93-1636

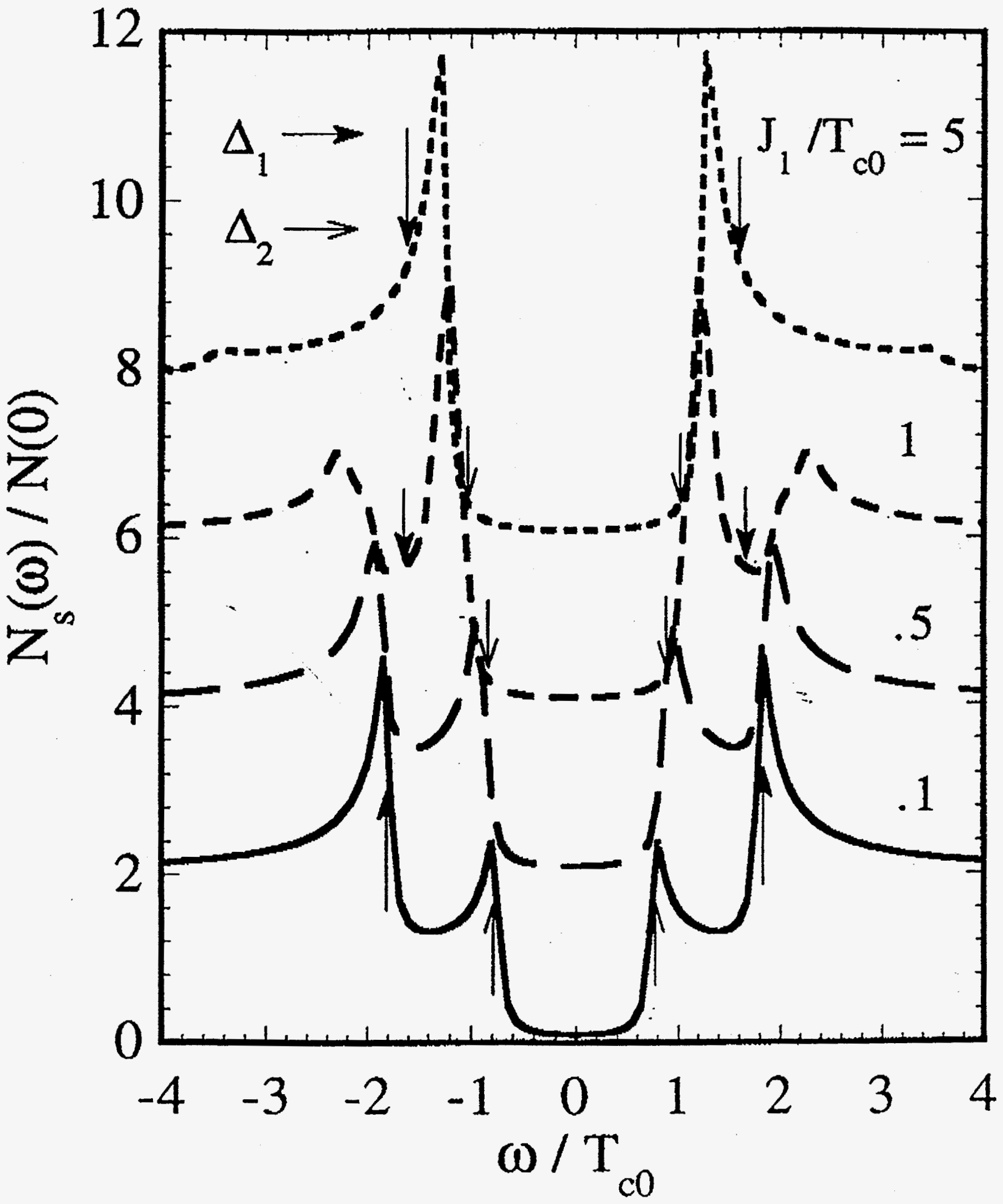

Fic. 3 
ORNL-DWG 93-1638

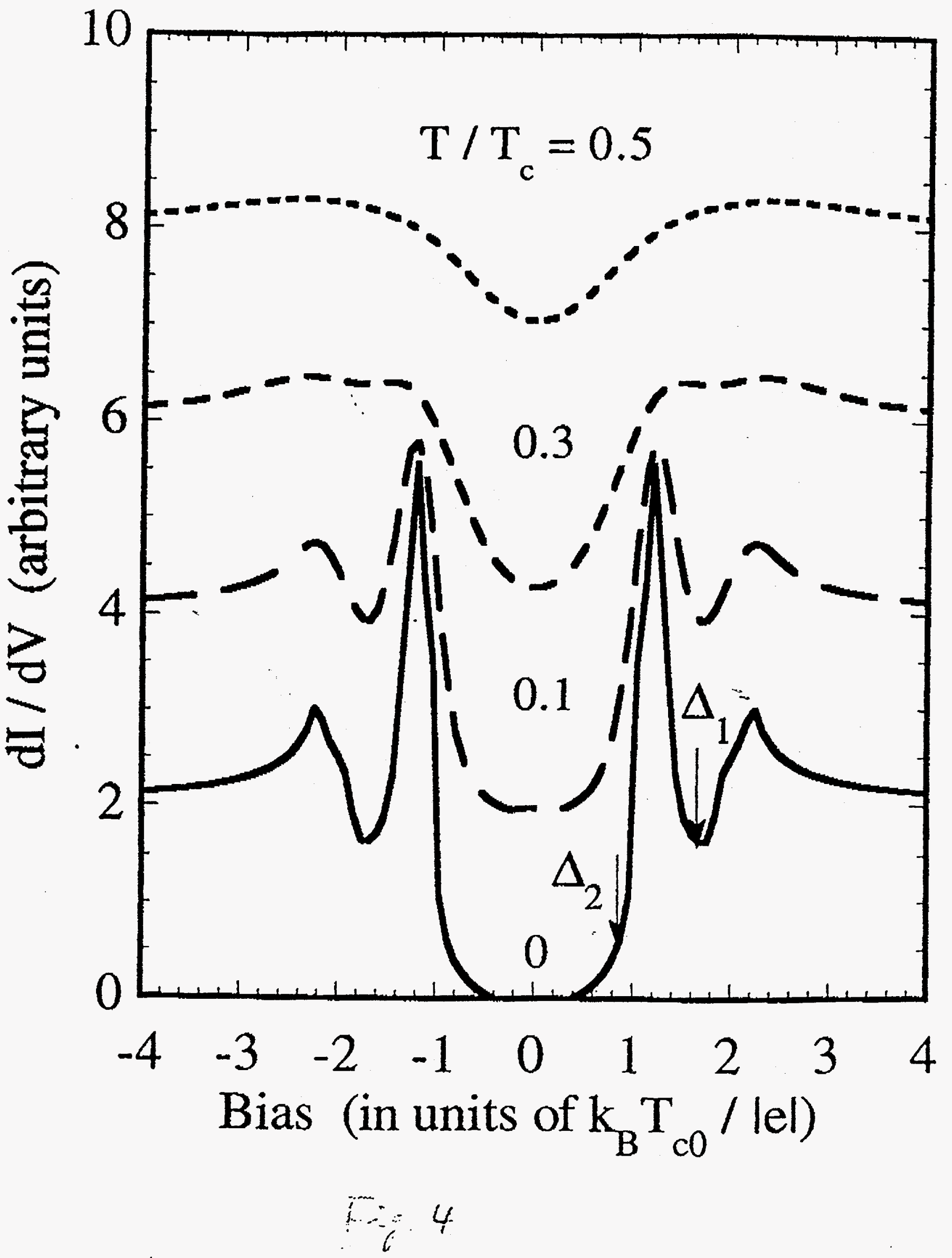


ORNL-DWG 93-1616

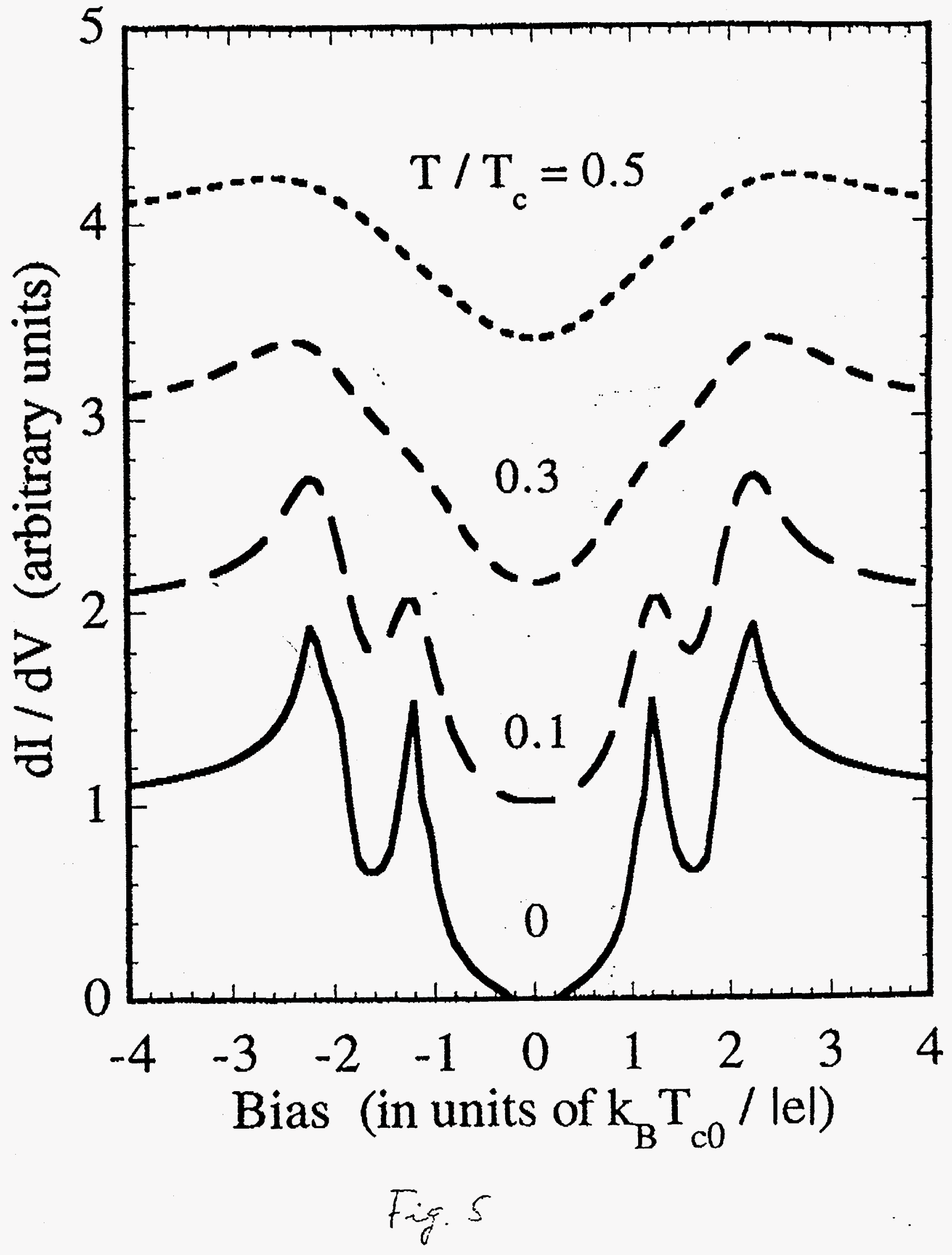


ORNL-DWG 93-1645

位

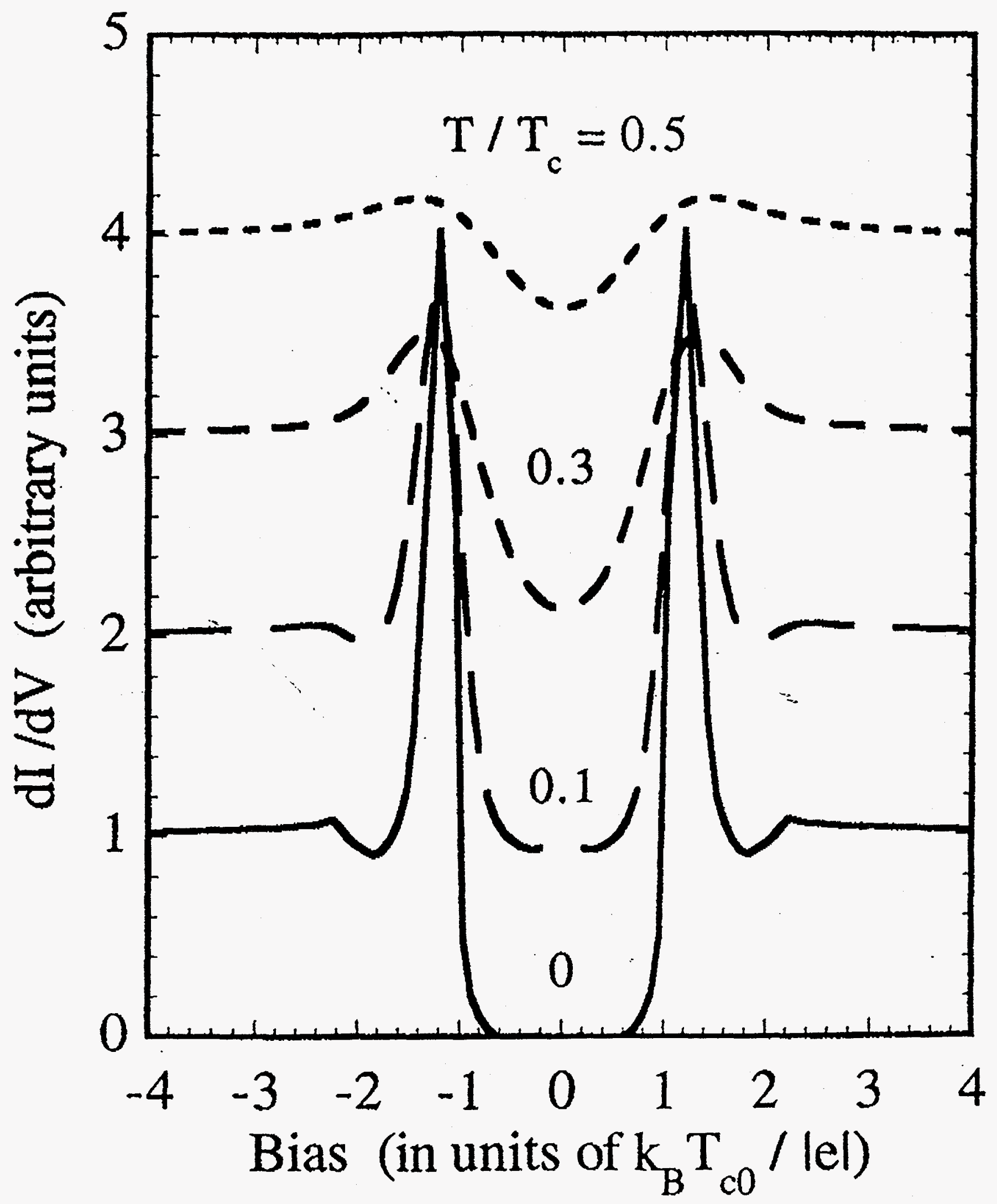

Fig 6 
'JUN-GI-1993' 14:12 FROM: SOLID STATE DIUISION/ORNL TO

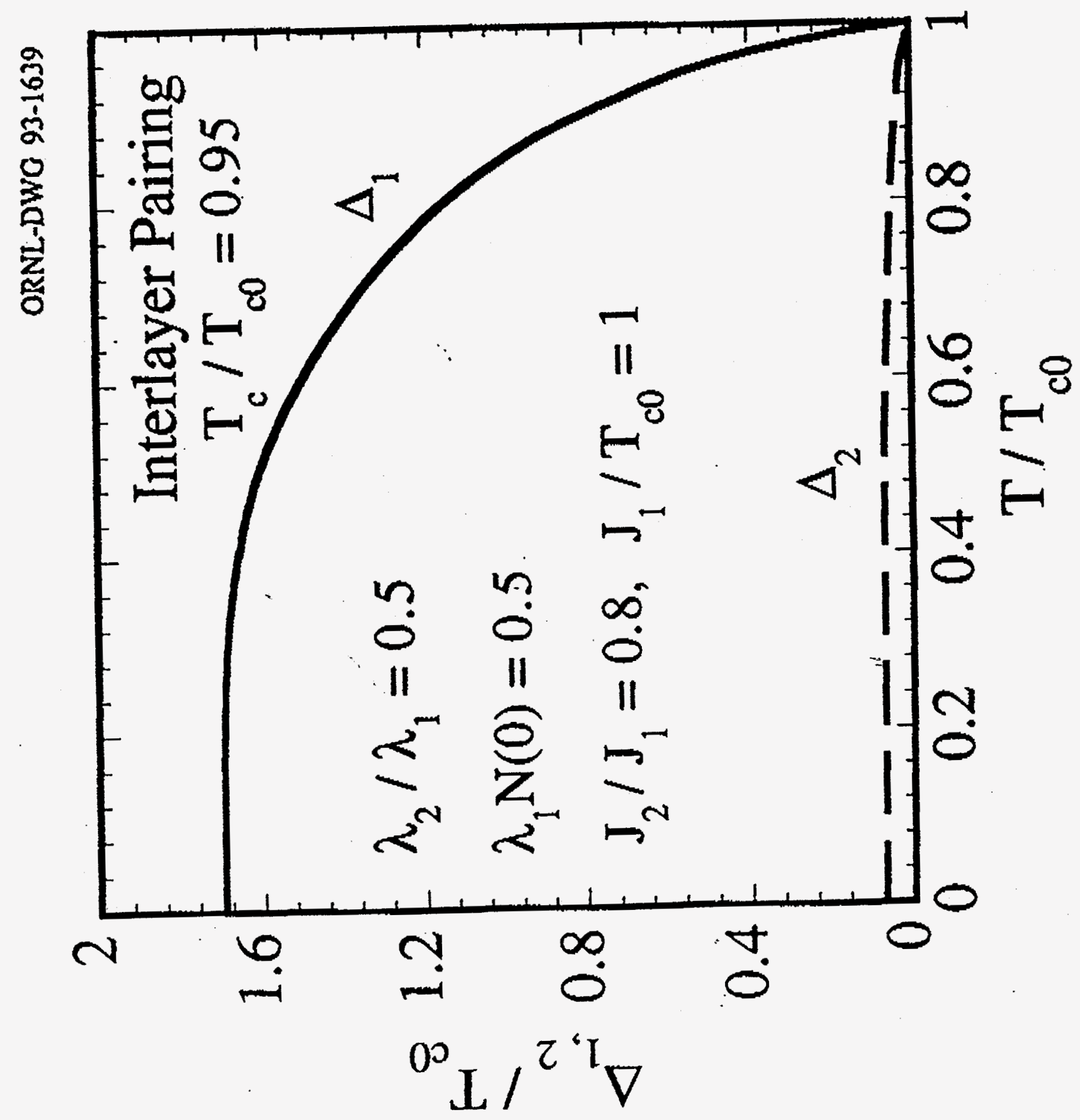

Fig. 7 


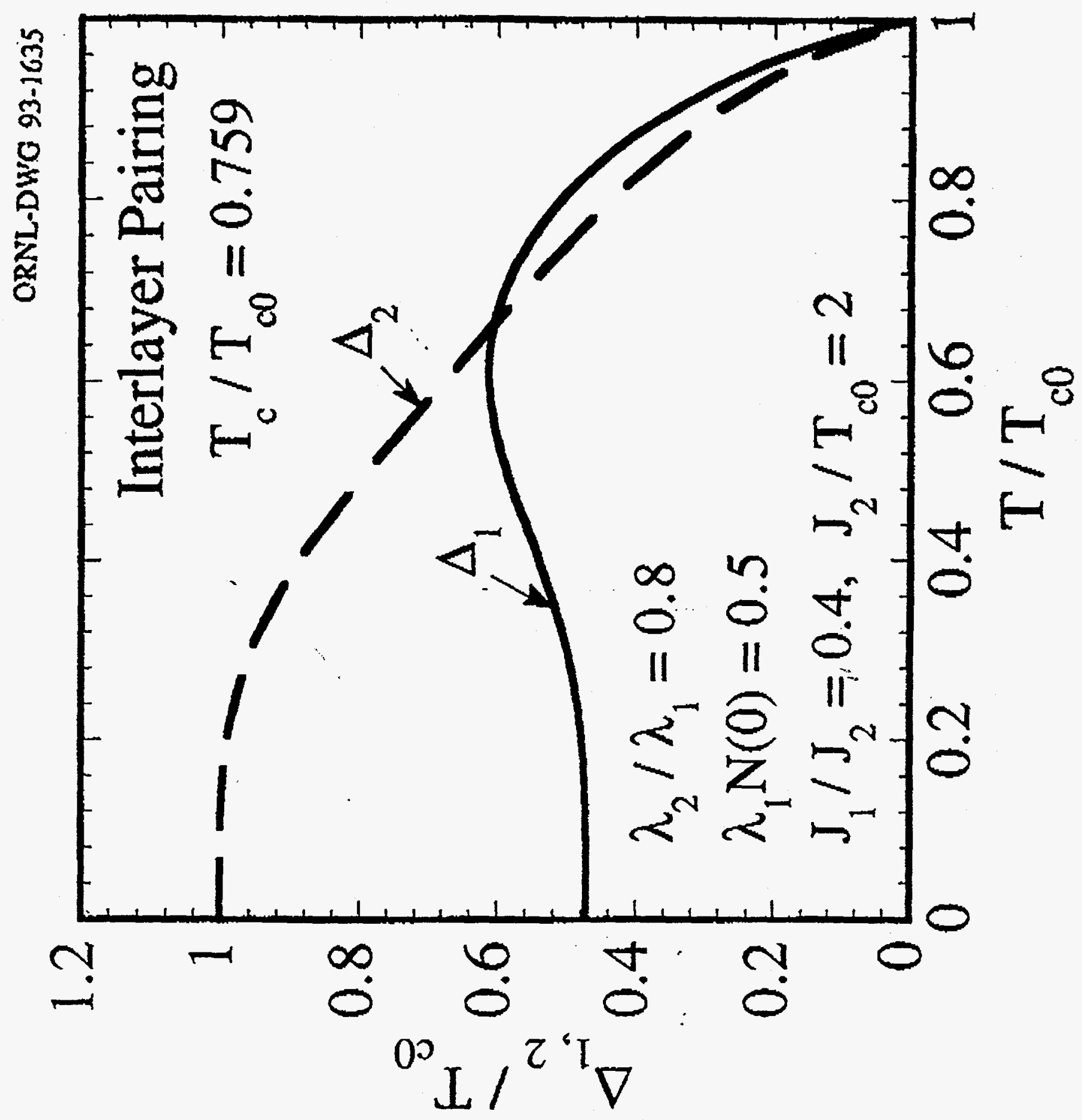

Fig. 8 
ORNL-DWG 93-1643R

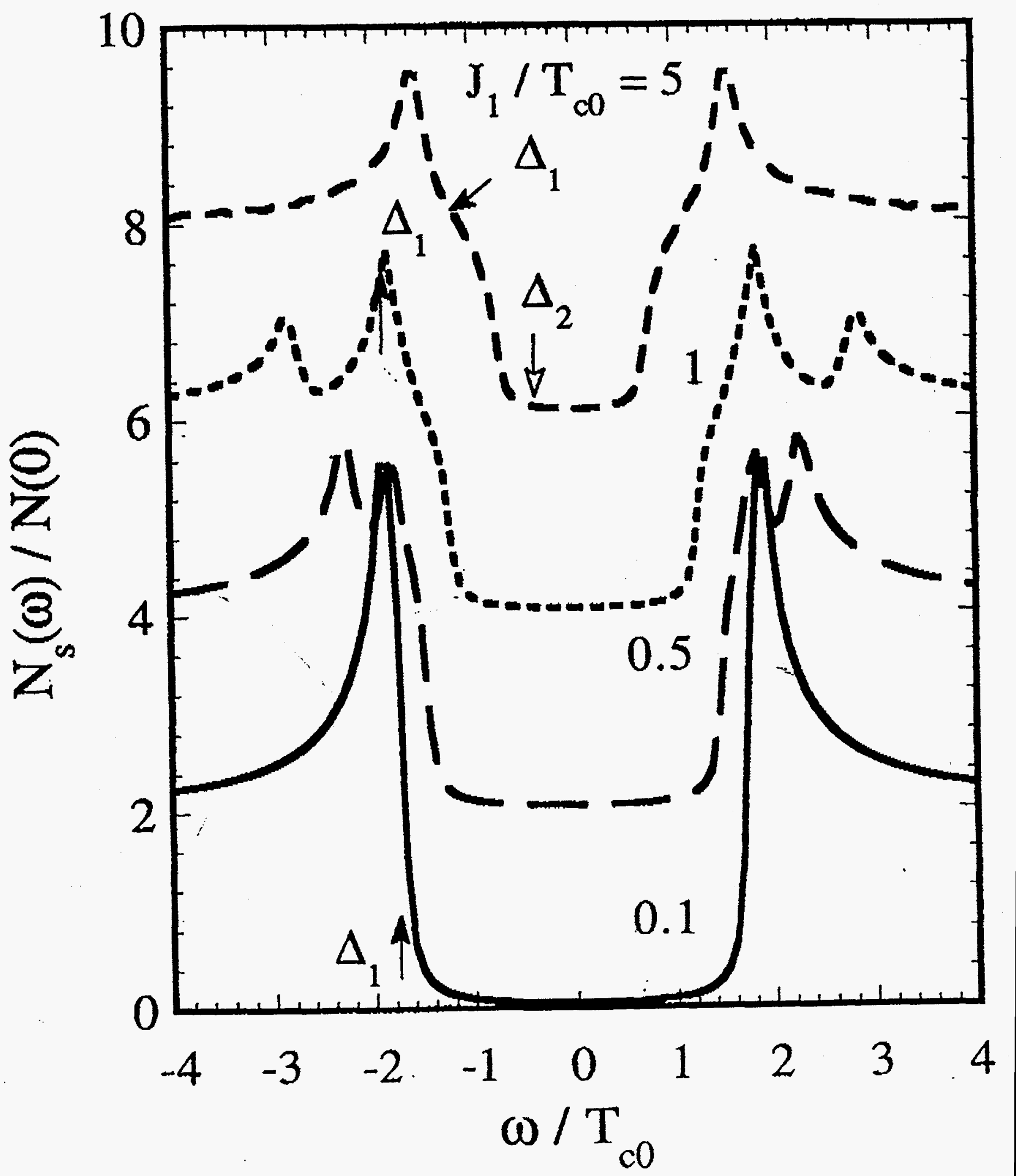

Fig. 9 
ORNL-DWG 93-1641R

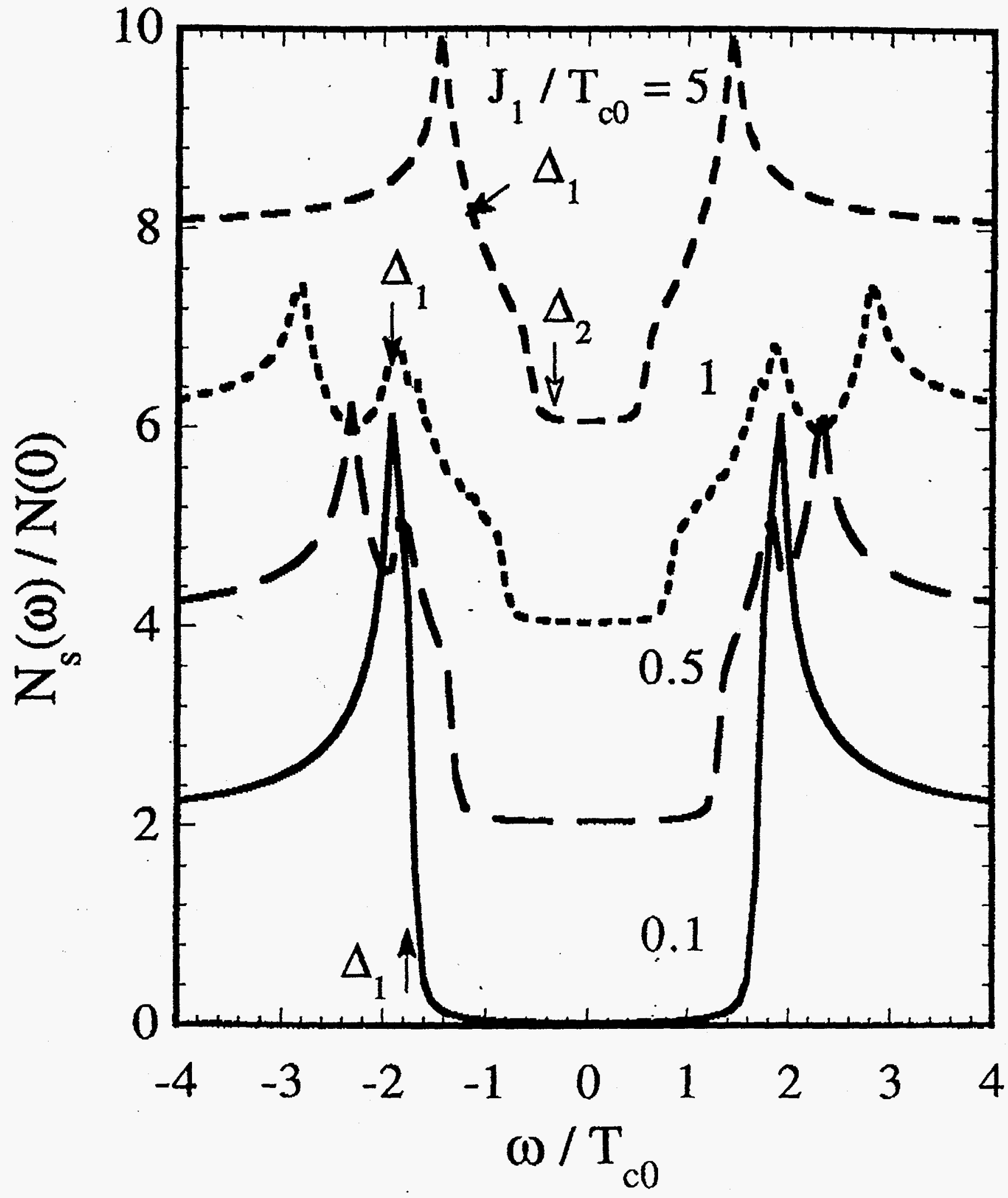

$F_{i j} 10$ 
ORNL-DWG 93-1644

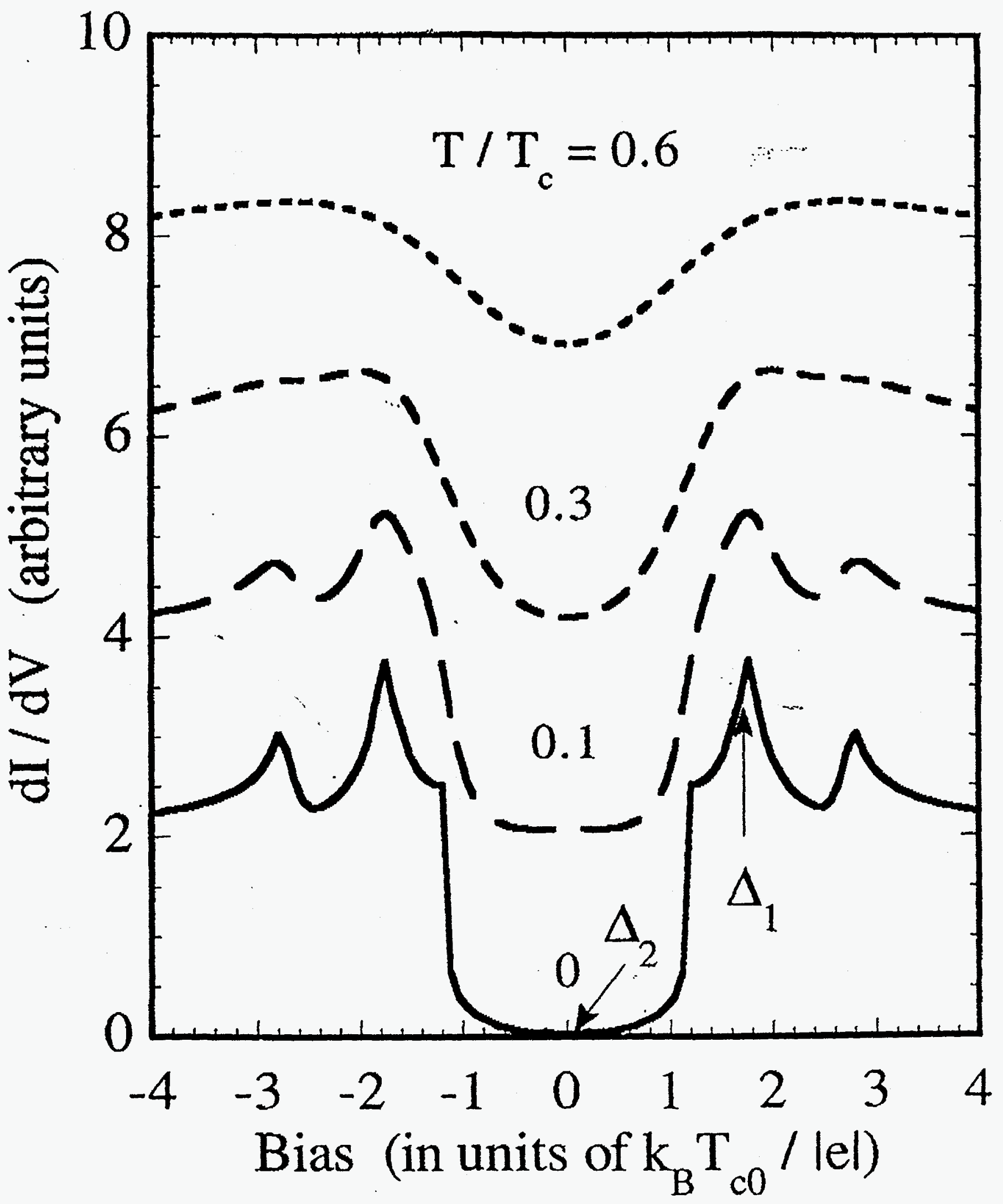

Fig. 11 